\title{
Proposed Vertical Expansion Tunnel
}

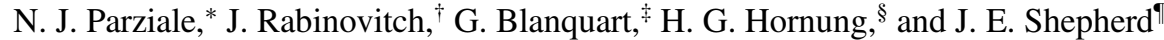 \\ California Institute of Technology, Pasadena, California 91125
}

\section{DOI: $10.2514 / 1 . J 052389$}

\begin{abstract}
It is proposed that the adverse effects from secondary diaphragm rupture in an expansion tunnel may be reduced or eliminated by orienting the tunnel vertically, matching the test gas pressure and the accelerator gas pressure, and initially separating the test gas from the accelerator gas by density stratification. This proposed configuration is termed the vertical expansion tunnel. Two benefits are 1) the removal of the diaphragm particulates in the test gas after its rupture, and 2) the elimination of the wave system that is a result of a real secondary diaphragm having a finite mass and thickness. An inviscid perfect-gas analysis and quasi-one-dimensional Euler computations are performed to find the available effective reservoir conditions (pressure and mass specific enthalpy) and useful test time in a vertical expansion tunnel for comparison to a conventional expansion tunnel and a reflected-shock tunnel. The maximum effective reservoir conditions of the vertical expansion tunnel are higher than the reflected-shock tunnel but lower than the expansion tunnel. The useful test time in the vertical expansion tunnel is slightly longer than the expansion tunnel but shorter than the reflected-shock tunnel. If some sacrifice of the effective reservoir conditions can be made, the vertical expansion tunnel could be used in hypervelocity ground testing without the problems associated with secondary diaphragm rupture.
\end{abstract}

\section{Nomenclature}

$a$
$E$
$e$
$h$
$L$

$\mathcal{P}$
$p$
$R$
$S$
$t$
$u$
$x_{1}$
$\gamma$
$\rho$
$\tau$
$S u b$

$=$ local speed of sound, $\mathrm{m} / \mathrm{s}$

$D \quad=$ binary diffusion coefficient, $\mathrm{m}^{2} / \mathrm{s}$

$E \quad=$ total energy multiplied by area, $\mathrm{J} / \mathrm{m}$

$=$ total energy, $\mathrm{J} / \mathrm{m}^{3}$

$h \quad=$ mass specific enthalpy, $\mathrm{MJ} / \mathrm{kg}$

$L \quad=$ length, $\mathrm{m}$

mw $=$ molecular weight

$\mathcal{P} \quad=$ pressure multiplied by cross-sectional area, $\mathrm{N}$

$p \quad=$ pressure, $\mathrm{Pa}$

$\mathcal{R}=$ mass density multiplied by cross-sectional area, $\mathrm{kg} / \mathrm{m}$

$S \quad=$ cross-sectional area, $\mathrm{m}^{2}$

$=$ time, $\mathrm{s}$

$u=$ velocity in $x_{1}$ direction, $\mathrm{m} / \mathrm{s}$

$x_{1}, x_{2}, x_{3}=$ orthogonal coordinate directions

$=$ ratio of specific heats

$\quad=$ mass density, $\mathrm{kg} / \mathrm{m}^{3}$

$=$ test time, $\mathrm{s}$

\section{Subscripts}

$\begin{array}{lll}A & = & \text { accelerator section } \\ \text { diff } & =\text { diffusion } \\ D & =\text { driver section }\end{array}$

Presented as Paper 2012-3263 at the 42nd AIAA Fluid Dynamics Conference and Exhibit, New Orleans, LA, 25-28 June 2012; received 13 October 2012; revision received 19 April 2013; accepted for publication 16 June 2013; published online 16 October 2013. Copyright ( 92013 by Nick Parziale. Published by the American Institute of Aeronautics and Astronautics, Inc., with permission. Copies of this paper may be made for personal or internal use, on condition that the copier pay the $\$ 10.00$ per-copy fee to the Copyright Clearance Center, Inc., 222 Rosewood Drive, Danvers, MA 01923; include the code 1533-385X/13 and $\$ 10.00$ in correspondence with the CCC

*Ph.D. Candidate, Graduate Aeronautical Laboratories, 1200 East California Boulevard, MC 205-45; currently Visiting Assistant Professor, Mechanical Engineering, Castle Point on Hudson, Hoboken, NJ 07030-5991 (Corresponding Author). Student Member AIAA.

${ }^{\dagger}$ Ph.D. Candidate, Graduate Aeronautical Laboratories, 1200 East California Boulevard, MC 205-45. Student Member AIAA.

¥Assistant Professor, Mechanical Engineering, 1200 East California Boulevard, MC 104-44. Member AIAA.

${ }^{\S}$ Professor Emeritus, Graduate Aeronautical Laboratories, 1200 East California Boulevard, MC 205-45. Fellow AIAA.

TProfessor, Graduate Aeronautical Laboratories, 1200 East California Boulevard, MC 205-45. Member AIAA.

$\begin{array}{ll}I & =\text { intermediate section } \\ i & =\text { state number } \\ N & =\text { nozzle section } \\ R & =\text { effective reservoir conditions }\end{array}$

\section{Introduction}

$\mathbf{F}$ IRST proposed by Trimpi [1] and Trimpi and Callis [2], the expansion tube and expansion tunnel (ET) have been developed as hypersonic ground-test facilities for approximately half a century. The appeal of an ET is the higher maximum reservoir mass specific enthalpy $h_{R}$ and reservoir pressure $p_{R}$ than in a reflected-shock tunnel (RST). The expanded parameter space in an ET is due to the unsteady manner in which the test gas is processed. Lukasiewicz [3] and Ben-Yakar and Hanson [4] describe the advantages and disadvantages of using a reflected-shock tunnel versus an expansion tunnel. Successful operation of an expansion tube or tunnel is often hampered by excessive perturbations in the test gas; efforts to reduce these perturbations are critical.

In Fig. 1, each numbered box represents a state in the expansion tunnel. Shock waves (SWs) are shown as thick solid lines. Expansion characteristics are shown as thinner solid lines. The contact discontinuities (CDs) are shown as dashed lines. A particle path (PP), representative of the test gas, is shown as a dashed-dot line. $L_{D}, L_{I}$, $L_{A}$, and $L_{N}$ are the lengths of the driver tube, the intermediate tube, the accelerator tube, and the nozzle, respectively.

An expansion tunnel operates ideally as follows: a pressure difference between the driver tube and the intermediate tube is prescribed, and the primary diaphragm is instantly ruptured. The primary contact surface impulsively advances from the primary diaphragm station into the intermediate tube. The impulsive advance of the primary contact surface necessitates a pressure discontinuity that processes the test gas (the primary shock wave). Upon arrival of the primary shock wave at the secondary diaphragm station, the secondary diaphragm instantly ruptures, and a secondary contact discontinuity impulsively advances into the accelerator tube. The impulsive advance of the secondary contact surface necessitates a pressure discontinuity that processes the accelerator gas (the secondary shock wave). Concurrent with the secondary diaphragm rupture, an unsteady expansion (centered at the secondary diaphragm station) processes the test gas. The test gas is accelerated, first through this unsteady expansion and then, in the case of an expansion tunnel, through the diverging nozzle at the end of the accelerator tube.

In the 1960s and 1970s, several expansion tubes and tunnels were constructed, and results were reported with significant perturbations in the test flow [5-8]. The perturbations were likely the result of 


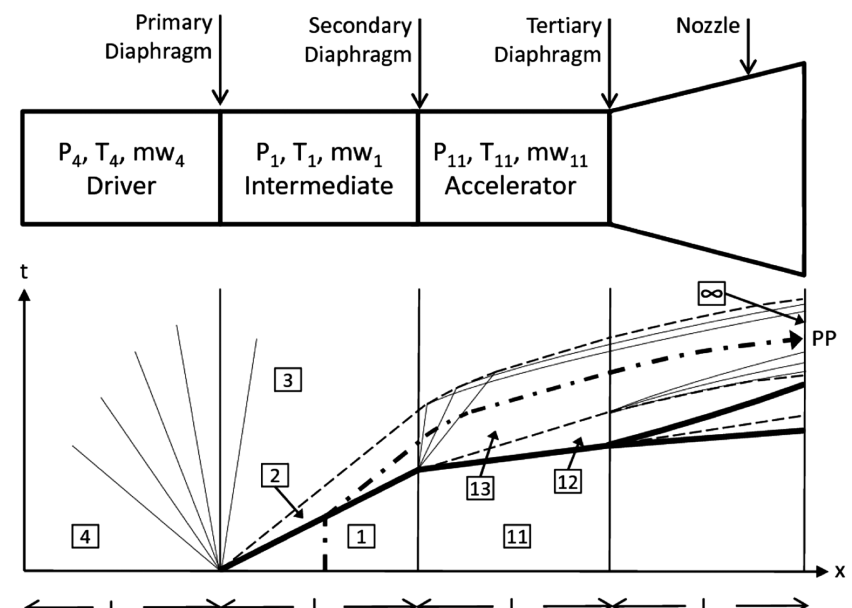

Fig. 1 Above is a schematic of an expansion tunnel. The states 4 , 1 , and 11 are the initial (or fill) conditions of the driver, the intermediate and the accelerator sections, respectively. Below is an $x$ - $t$ diagram of expansion tunnel operation.

acoustic waves in the driver gas being transmitted into the test gas and/or nonideal rupture of the secondary diaphragm.

The disruptive acoustic waves that are transmitted to the test gas from the driver gas occur for certain ratios of sound speed across the primary contact surface, $a_{3} / a_{2}[\underline{9}, 10]$. Jacobs [11] used numerical techniques to study the introduction of perturbations from the driver gas to the test gas. Mitigation of these unsteady sources of noise by appropriate design and operation of an expansion tube has been successfully demonstrated at the University of Illinois at UrbanaChampaign by Dufrene et al. [12].

The nonideal rupture of the secondary diaphragm can disturb the test gas in three ways: 1) the reduction in useful test time due to finite secondary diaphragm rupture duration $[13] ; 2$ ) the wave system that arises from the reflection of the primary shock wave off the secondary diaphragm can affect the thermo-chemical properties of the gas [14]; and 3) the diaphragm particulates can contaminate the test gas by introduction of foreign matter to the test flow, reacting with the test gas if the temperature is sufficiently high.

Since the inception of the simple shock tube, significant efforts have been made to understand and mitigate diaphragm rupture issues [15-19]. Researchers have extended this basis of knowledge to the problems associated with secondary diaphragm rupture in an expansion tube [20-22]. Furthermore, models of the secondary diaphragm rupture process have been formulated and can be found in the literature $[14,23]$. The particulates, which travel on the order of the test flow velocity, can also impact and damage the test article [21].

A number of expansion tube/tunnel facilities exist, including the $\mathrm{X}$ facilities at the University of Queensland [23], the HYPULSE facility at NASA [24], the JX-1 facility at Tohoku University [25], the 6 in. expansion tube at Stanford University $[4,26]$, the HET facility at University of Illinois at Urbana-Champaign [12], and the LENS X facility at CUBRIC [27]. These facilities have been used successfully for hypersonic aerodynamics and combustion research. Still, some of the proceedings and articles show results from these facilities with significant test gas perturbations (often conveyed through pressure measurements). In particular, many of these perturbations appear in the vicinity of the test gas/accelerator gas interface; this is evidence of the secondary diaphragm rupture adversely affecting the results.

In this work, we propose a means of mitigating the effects of secondary diaphragm rupture in an expansion tunnel by eliminating the need for a secondary diaphragm. The secondary diaphragm is no longer required if the facility is oriented vertically and the intermediate and accelerator tubes are filled to the same initial pressure with gases of different density, light over heavy (e.g., helium over air). The intermediate/accelerator interface would be initially separated by a fast-acting sliding valve, allowing the facility to be filled with the accelerator gas and test gas and reduce the diffusion across the interface. The fast-acting valve will be removed before the bursting of the primary diaphragm, leaving the interface hydrodynamically stable. This facility is termed the vertical expansion tunnel (VET). A comparison of the available parameter space in a vertical expansion tunnel (VET), an expansion tunnel (ET), and a reflected-shock tunnel (RST) is presented. The comparison is restricted to perfect-gas conditions. Perfect-gas quasi-one-dimensional Euler computations are used to calculate the available test time in the VET and the ET; in addition, a referenced method is used to calculate the test time in an RST. It is found that the maximum reservoir mass specific enthalpy and pressure of the VET is above the RST but below the ET; the test time in the VET is slightly longer than the ET but shorter than the RST.

\section{Calculation of Available Conditions}

In this section, a comparison of the parameter space available in a vertical expansion tunnel (VET), conventional expansion tube (ET), and reflected-shock tunnel (RST) is presented. The driver pressure $\left(p_{4}=8.16 \mathrm{MPa}\right)$ is chosen so that it could be filled by conventional research He gas bottles. In all but one case, the test gas temperature is restricted to be below $\approx 2000 \mathrm{~K}$ to ensure a fair comparison between facilities and avoid the detrimental effects of test gas heating [13]. The restriction of maximum test gas temperature permits the perfectgas assumption. Additionally, at this pressure ratio $\left(p_{4} / p_{1}\right)$, the sound speed ratio is $a_{3} / a_{2} \approx 0.57$; at this $a_{3} / a_{2}$, Dufrene et al. [12] observed experimentally that the perturbations of the Paull and Stalker type [10] in the freestream were acceptable. To aid in comparison, the test gas in each facility is expanded to a freestream Mach number of 5.5. In the VET, this necessitates the use of a nozzle at the end of the expansion tube to increase the Mach number, making it an expansion tunnel. A nozzle is not needed at the end of the conventional ET because of the more efficient unsteady expansion.

Pressure-velocity diagrams are used to find the conditions of the test gas as it is processed by the wave systems (for reference, follow the particle path, PP, in Fig. 1). The static pressure and velocity must be matched in states 2 and 3 and in states 12 and 13. This is done by plotting the expansion

$$
\frac{p_{3}}{p_{4}}=\left(1-\frac{\left(\gamma_{4}-1\right)\left(u_{3}-u_{4}\right)}{2 a_{4}}\right)^{\frac{2 \gamma_{4}}{\gamma_{4}-1}}
$$

and shock relationships

$$
\frac{u_{2}-u_{1}}{a_{1}}=\frac{p_{2}-p_{1}}{\gamma_{1} p_{1} \sqrt{1+\frac{\left(\gamma_{1}+1\right)\left(p_{2}-p_{1}\right)}{2 \gamma_{1} p_{1}}}}
$$

in pressure-velocity space and finding the point of intersection [28]. Here, $\gamma$ is the ratio of specific heats, $p$ is the static pressure, $u$ is the velocity, and $a$ is the sound speed. Equations (1) and (2) are annotated for finding the conditions after primary diaphragm rupture and are

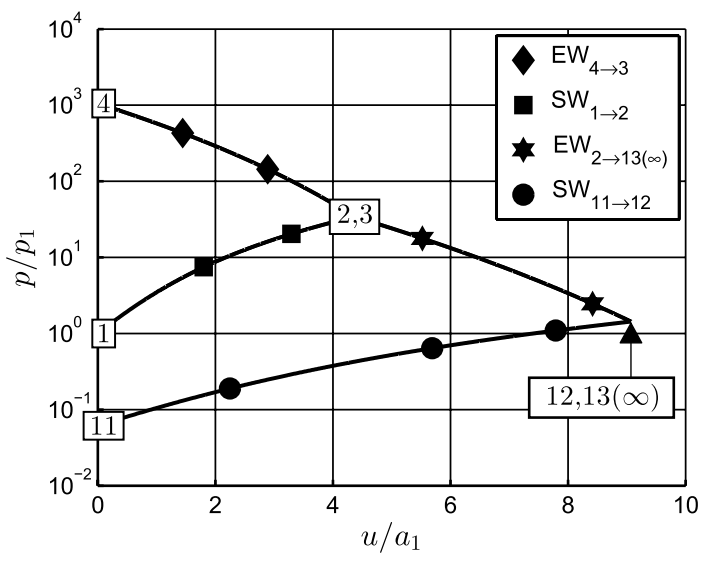

Fig. 2 Pressure-velocity diagram for a conventional expansion tube. Expansion waves are denoted by "EW", and shock waves are denoted by "SW". Note that " $13(\infty)$ " denotes the freestream state. 


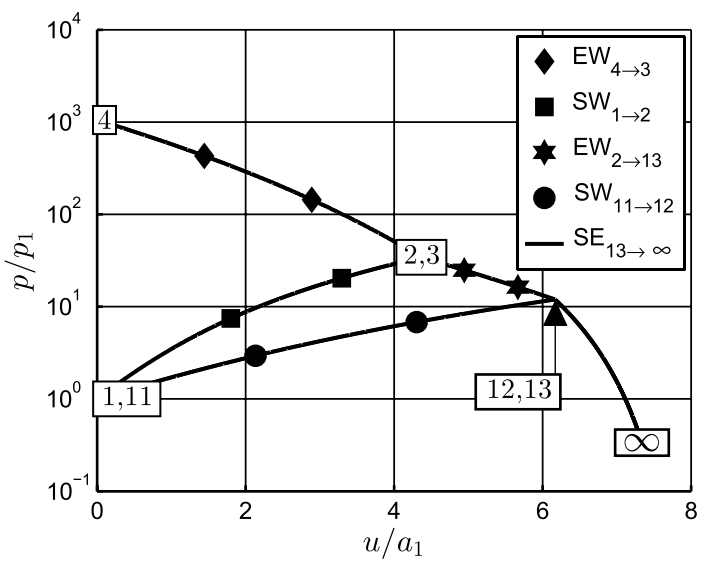

Fig. 3 Pressure-velocity diagram for a vertical expansion tunnel. Expansion waves are denoted by "EW", shock waves are denoted by "SW", and the steady expansion is denoted by "SE". Note that " $\infty$ " denotes the freestream state.

analogous to the equations that would be used to evaluate states 12 and 13. In the VET, the gas from state 13 is expanded through a nozzle using the usual steady quasi-one-dimensional gas-dynamic equations.

In a conventional ET, a diaphragm is located between the intermediate and accelerator chambers so that there can be a mismatch of the fill pressures in states 1 and 11 (pressure-velocity diagram in Fig. 2). This secondary diaphragm may be eliminated by orienting the tunnel vertically, matching the initial test gas pressure and the accelerator gas pressure, and initially separating the test gas from the accelerator gas by density stratification (pressure-velocity diagram in Fig. 3). The unsteady expansion centered at the secondary diaphragm station is stronger in the ET when compared with the VET; for this reason, the conventional ET is able to reach higher effective reservoir states than the VET. If some sacrifice of the effective reservoir conditions can be made, the VET can be used in hypervelocity ground testing without the problems associated with secondary diaphragm rupture.

The available conditions and test times for a given set of fill pressures are tabulated in Table 1 for three types of impulse hypersonic facilities, the VET, the ET, and the RST. In Table $\underline{1}, p_{4}$, $p_{1}$, and $p_{11}$ are the initial pressures of the driver, intermediate, and accelerator sections, respectively; $h_{R}$ and $p_{R}$ are the effective reservoir mass specific enthalpy and pressure; $T_{\mathrm{Max}}$ is the maximum temperature the test gas is raised to in the facility; $u_{\infty}, p_{\infty}, \rho_{\infty}, T_{\infty}$, and $M_{\infty}$ are the freestream velocity, pressure, density, temperature, and Mach number, respectively; and $\tau$ is the test time. The effective reservoir conditions (reservoir pressure and mass specific enthalpy) for the conventional ET and the VET are found by isentropic compression of state 13 to rest. The pressure $p_{1}$ for the first shock tunnel case (RST-1) is chosen such that it is operated in the tailored mode. The pressure $p_{1}$ for the second shock tunnel case (RST-2) is chosen so that the temperature in the test gas does not exceed $2000 \mathrm{~K}$. At this pressure ratio $\left(p_{4} / p_{1}\right)$, the RST will be operated in an overtailored mode, and the Mach number of the primary shock is $10 \%$ higher in RST-2 relative to the tailored condition (RST-1). The pressure $p_{1}$ for the third shock tunnel case (RST-3) is chosen such that the reservoir mass specific enthalpy is matched to the VET case and required a 50\% increase in the Mach number of the primary shock relative to the tailored condition (RST-1). In this case, the test gas will be reacting, and so Cantera [29] with the Shock and Detonation Toolbox [30] is used to evaluate the conditions in the reservoir and through the nozzle. The appropriate thermodynamic data $[\underline{31}, \underline{32}]$ and reaction rates [33] are found in the literature. The test gas is assumed to be in chemical equilibrium in the reservoir and up to the throat of the nozzle. The run conditions at the nozzle exit are found by the integration of a system of coupled ordinary differential equations (accounting for finite-rate chemistry) from the throat to the nozzle exit; the equations are derived in [34]. At matched reservoir mass specifc enthalpy, the VET has a higher effective reservoir pressure than in the RST-3 case. This performance advantage of the VET relative to the RST would become increasingly apparent by increasing the local Mach number in state 2 because the total temperature and pressure gain in an unsteady expansion varies strongly with Mach number. In the RST-3 case, there is 3.5\% NO (by mole) in the freestream; all other cases in all facilities produce a negligible amount of NO.

If the quantity of interest in ground-test facilities is effective reservoir conditions, then the capability of the VET is above the RST but below the ET. One advantage of the ET or VET over the shock tunnel is a lower maximum test gas temperature for a given reservoir mass specific enthalpy, and so the detrimental effects of the test gas being partially dissociated and partially vibrationally excited are less severe. To increase test time, one can scale the facility size up; however, facility wear in an RST, particularly nozzle throat heating, will become problematic at lower effective reservoir conditions than in the ET or VET [13].

\section{Test-Time Calculation for the Reflected-Shock Tunnel and Expansion Tunnel}

Test-time calculations for the facilities shown in Table 1 require facility sizing choices to be made. An $L / d(1.27 \mathrm{~m} / 25.4 \overline{\mathrm{mm}})$ ratio of 50 was chosen to minimize the effects of the boundary layer on the walls of the shock tube [35] and is held constant for the RST, ET, and VET for comparison. The overall length was chosen so that it may fit into a single-story lab as a demonstrator-type facility. It is important to note that increasing the test time of the facility simply requires an increase in the facility length, noting that the test time scales approximately linearly with combined length of the intermediate and accelerator sections.

In the RST, a 10 deg half-angle nozzle of throat diameter $8.46 \mathrm{~mm}$ (1/3 in.), length $175 \mathrm{~mm}$, and area ratio 70 is chosen so that the test section is of similar size to the VET. Test time (listed in Table 1) was considered to begin after the nozzle startup time (estimate formulated by Smith [36]) and end after the driver gas contaminates the test gas (estimate formulated by Davies and Wilson [37]). This methodology to estimate the test time has been successfully demonstrated by Sudani and Hornung [38].

When calculating the maximum test time for a given total tube length in a conventional ET, the lengths for the intermediate and accelerator sections must be optimized. The sum of their lengths is subject to the $L / d$ constraint given previously. Following [12,39], the calculated test time is the time between the secondary contact discontinuity and intersection of the tail and the reflected head of the secondary expansion wave (Fig. 4). If the test section is not located at the ideal spatial location, the test time will be limited by the arrival of the head or reflected tail of the secondary expansion wave. With $L / d$ limited to 50, the ideal lengths for the intermediate and accelerator tubes are determined to be 0.86 and $0.41 \mathrm{~m}$, respectively, for the conditions given in Table 1 . In the absence of a nozzle, the same

Table 1 Comparison of run conditions available for RST, ET, and VET

\begin{tabular}{|c|c|c|c|c|c|c|c|c|c|c|c|c|}
\hline & $p_{4}(\mathrm{He}), \mathrm{MPa}$ & $p_{1}$ (air), $\mathrm{kPa}$ & $p_{11}(\mathrm{He}), \mathrm{kPa}$ & $h_{R}, \mathrm{MJ} / \mathrm{kg}$ & $p_{R}, \mathrm{MPa}$ & $T_{\text {Max }}, \mathrm{K}$ & $u_{\infty}, \mathrm{km} / \mathrm{s}$ & $p_{\infty}, \mathrm{kPa}$ & $\rho_{\infty}, \mathrm{kg} / \mathrm{m}^{3}$ & $T_{\infty}, \mathrm{K}$ & $M_{\infty}$ & $\tau, \mu s$ \\
\hline ET & 8.16 & 7.75 & 0.47 & 5.7 & 11 & 1980 & 3.1 & 11 & 0.048 & 800 & 5.5 & 56 \\
\hline VET & 8.16 & 7.75 & 7.75 & 3.8 & 2.4 & 1980 & 2.5 & 2.6 & 0.017 & 534 & 5.5 & 82 \\
\hline RST-1 & 8.16 & 106 & -- & 1.8 & 7.8 & 1780 & 1.8 & 8.4 & 0.116 & 252 & 5.5 & 6400 \\
\hline RST-2 & 8.16 & 77.0 & -- & 2.0 & 6.7 & 2010 & 1.9 & 7.3 & 0.089 & 285 & 5.5 & 5700 \\
\hline RST-3 & 8.16 & 7.10 & -- & 3.8 & 2.0 & 3110 & 2.5 & 0.9 & 0.008 & 553 & 5.5 & 920 \\
\hline
\end{tabular}




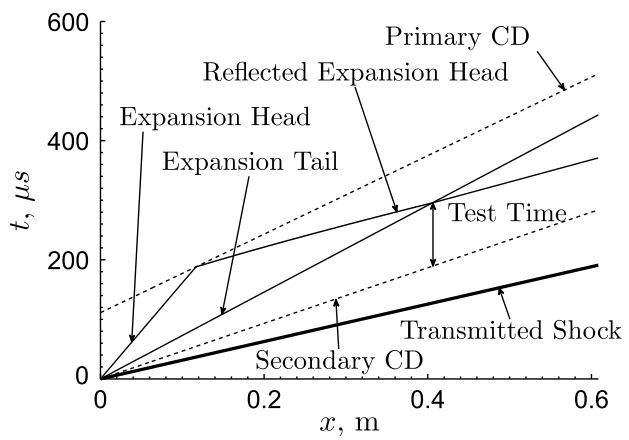

Fig. 4 Schematic $x$ - $t$ diagram for a conventional ET, focusing on the accelerator tube, and the ideal test time.

methodology can be used to evaluate the test time in a VET. In this case, the test time is estimated to be $106 \mu \mathrm{s}$.

\section{Test-Time Calculation for the Vertical Expansion Tunnel}

Calculation of test time in the VET requires consideration of the location and length of the nozzle, both of which significantly affect the test time. Unfortunately, no analytical results are readily available when a nozzle is present. To explore a large parameter space at a relatively low computational cost, quasi-one-dimensional Euler computations are performed based on a method suggested by Glaister [40]. A brief summary of this method is presented next, followed by the results.

\section{A. Numerical Methods}

In three dimensions, the Euler equations are given by Eqs. (3-5). Combining these with the equation of state for an ideal gas [Eq. (6)], allows for the solution of an inviscid unsteady compressible flowfield to be calculated:

$$
\begin{gathered}
\rho_{t}+\nabla \cdot(\rho \mathbf{u})=0 \\
(\rho \mathbf{u})_{t}+\nabla \cdot(\rho \mathbf{u u})=-\nabla p \\
e_{t}+\nabla \cdot[\mathbf{u}(e+p)]=0 \\
e=\frac{p}{\gamma-1}+\frac{1}{2} \rho \mathbf{u} \cdot \mathbf{u}
\end{gathered}
$$

Here, $\rho=\rho(\mathbf{x}, t), p=p(\mathbf{x}, t), e=e(\mathbf{x}, t)$, and $\mathbf{u}=\mathbf{u}(\mathbf{x}, t)=$ $\left[u_{1}(\mathbf{x}, t), u_{2}(\mathbf{x}, t), u_{3}(\mathbf{x}, t)\right]^{T}$ represent the density, pressure, total energy, and the three components of velocity, respectively, at a general position in space given by $\mathbf{x}=\left(x_{1}, x_{2}, x_{3}\right)^{T}$ at time $t$.

Assuming an ideal nozzle (small change in area with respect to distance), all changes in the flow depend only on one coordinate direction. Following the analysis of Glaister [40], the compressible Euler equations for duct flow can be rewritten as

$$
\left(\begin{array}{c}
\mathcal{R} \\
\mathcal{R} u \\
E
\end{array}\right)_{t}+\left(\begin{array}{c}
\mathcal{R} u \\
\mathcal{P}+\mathcal{R} u^{2} \\
u(E+\mathcal{P})
\end{array}\right)_{x_{1}}=\left(\begin{array}{c}
0 \\
\mathcal{P} \frac{S^{\prime}\left(x_{1}\right)}{S\left(x_{1}\right)} \\
0
\end{array}\right)
$$

with

$$
E=\frac{\mathcal{P}}{\gamma-1}+\frac{1}{2} \mathcal{R} u^{2}
$$

Here, it is assumed that all variables are only a function of $x_{1}$ (direction along the nozzle centerline), and $S\left(x_{1}\right)$ represents the cross-sectional area at a point $x_{1}$. A new set of "conserved" variables
$\mathcal{R}, E$, and $\mathcal{P}$ have been used, where $\mathcal{R}=S\left(x_{1}\right) \rho, E=S\left(x_{1}\right) e$, and $\mathcal{P}=S\left(x_{1}\right) p$. The resulting equations are very similar to the original Euler equations, with the addition of a source term, which is seen on the right hand side of Eq. (7).

A standard Roe Riemann solver [41] is used to solve Eqs. (7) and (8). The additional source term is taken into account by modifying the wave strengths in the Roe solver [40]. A higher-order flux correction is added to the Roe scheme using wave limiters to make the numerical scheme less diffusive [42]. A Van Leer limiting function is used for this flux correction [43]. Because of the high initial pressure ratio between the driver gas and intermediate gas $\left(p_{4} / p_{1} \approx 1000\right)$, steep gradients in fluid properties and rapidly changing eigenvectors are created. This motivated the choice of a robust limiting function originally proposed by Lax and Liu [44], which is designed to work with systems of nonlinear equations. The flux limiters are not used in the vicinity of walls or right at the interface between two fluids with different values of $\gamma$. Last, the entropy fix proposed by Sanders et al. [45] is implemented, and no entropy violating solutions are observed.

This limited Roe scheme is combined with a method proposed by Abgrall and Karni [46] to treat the interfaces between gases with different $\gamma$. Two separate fluxes are used for the same face between neighboring cells, which prevents any unphysical oscillations from occurring at the fluid interface. Although the scheme no longer conserves total energy, a detailed analysis was performed by Abgrall and Karni, showing how offsetting errors are induced. Furthermore, it has been checked that the total energy loss in the computations presented in this investigation are negligible $<.001 \%$. Finally, a simple advection equation with a passive scalar is used to track the fluid interfaces and is solved using a modified semi-Lagrangian scalar scheme [47].

\section{B. Numerical Results: Verification}

A one-dimensional (1-D) perfect-gas Euler computation with the same initial conditions as the proposed VET (see Table 1) was performed. In this simulation, $L_{D}=2.0 \mathrm{~m}, L_{I}=0.86 \mathrm{~m}$, and $L_{A}=1.0 \mathrm{~m}$. The subscripts correspond to the driver section, intermediate section, and accelerator section, respectively. The accelerator section is made longer than the theoretical necessary length of $0.41 \mathrm{~m}$ (see Sec. III) to ensure that the tail and reflected head of the secondary expansion intersect inside the computational domain, as sketched in Fig. 4 . The primary diaphragm is located at $x=0 \mathrm{~m}$, and the interface between the intermediate and accelerator sections is at $x=0.86 \mathrm{~m}$.

To visualize the results of the 1-D perfect-gas Euler computations, a numerical $x$ - $t$ diagram is made using a numerical schlieren method, where contours of the function $-\log \left(\left|\partial \rho / \partial x_{1}\right|\right)$ are plotted (Fig. 5). The simulation results capture the theoretical wave system depicted in Fig. 1. There is also good quantitative agreement between the

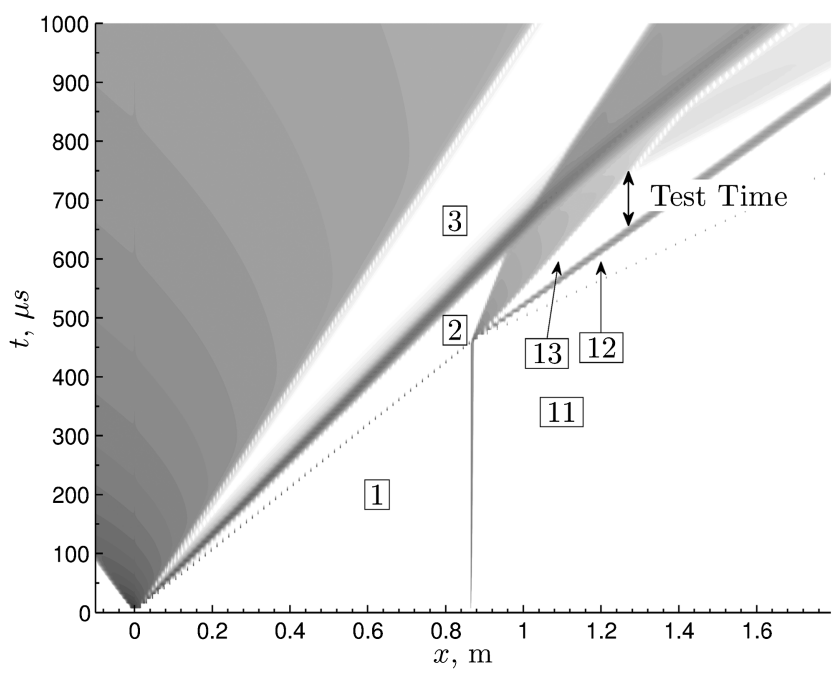

Fig. 5 Numerical $x$ - $t$ diagram for a one-dimensional perfect gas Euler simulation with the same initial conditions as the proposed VET. 


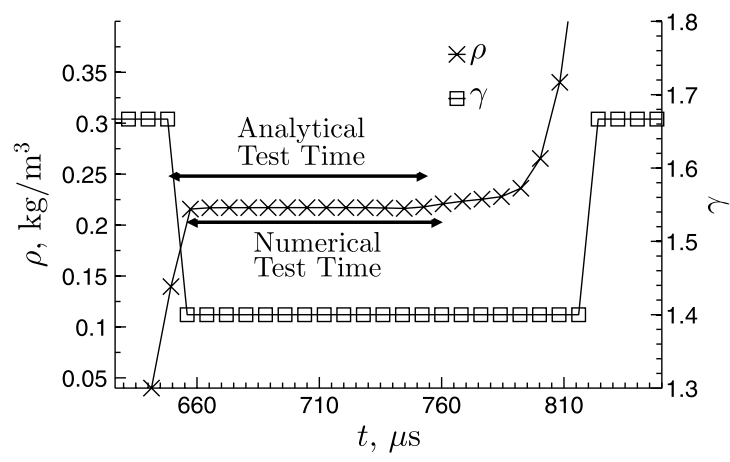

Fig. 6 Comparison between the analytic test time and the numerical test time for the 1-D case.

numerical results and the analytical solution. The numerical results differ from the analytical results by no more than the third significant digit $(\approx 0.5 \%)$ for pressure, velocity, or density in states 2 and 3 and states 12 and 13. The numerical values have been averaged over the respective appropriate section, at $t=450 \mu$ s for states 2 and 3 and at $t=600 \mu$ s for states 12 and 13 .

Comparison of the test time between the 1-D perfect-gas Euler simulations and the analytic calculations is also necessary to fully validate the numerical technique. The numerical test time is defined to be when the density is within $1 \%$ of the average value of density in the constant region, state 13 . Figure 6 shows a comparison between the analytic test time and the numerical test time. Although fluctuations during the test time in an experimental facility are expected to be larger than $1 \%$, we note that these analyses are for validation of the numerical technique.

The analytical test time is calculated to be $106 \mu \mathrm{s}$; the numerical test time is found to be $104 \mu$ s. Figure 6 shows that the test times are also slightly offset in time from each other. This discrepancy is due to the numerical methods used. Contact discontinuities, which are infinitely thin in the ideal case, are spread out over a few cells in simulations due to numerical diffusion. In addition, the reflection of waves off of a contact discontinuity of finite thickness may introduce errors in the simulations. Nevertheless, these small differences were acceptable, and so quasi-1-D Euler simulations were started with the addition of the nozzle at the end of the accelerator tube.

\section{Vertical Expansion Tunnel with Nozzle}

The addition of a nozzle at the end of the accelerator tube expands the design parameter space that one must investigate. In this analysis, we consider changes to the location of the nozzle and lengths of the

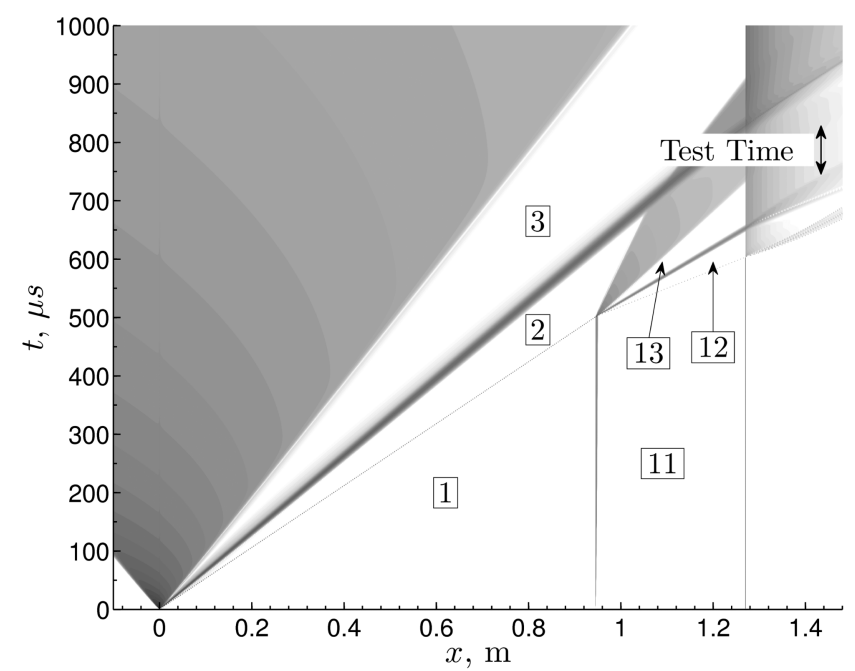

Fig. 7 Numerical $x$ - $t$ diagram for an expansion tunnel with a $10 \mathrm{deg}$ half-angle diverging conical nozzle.

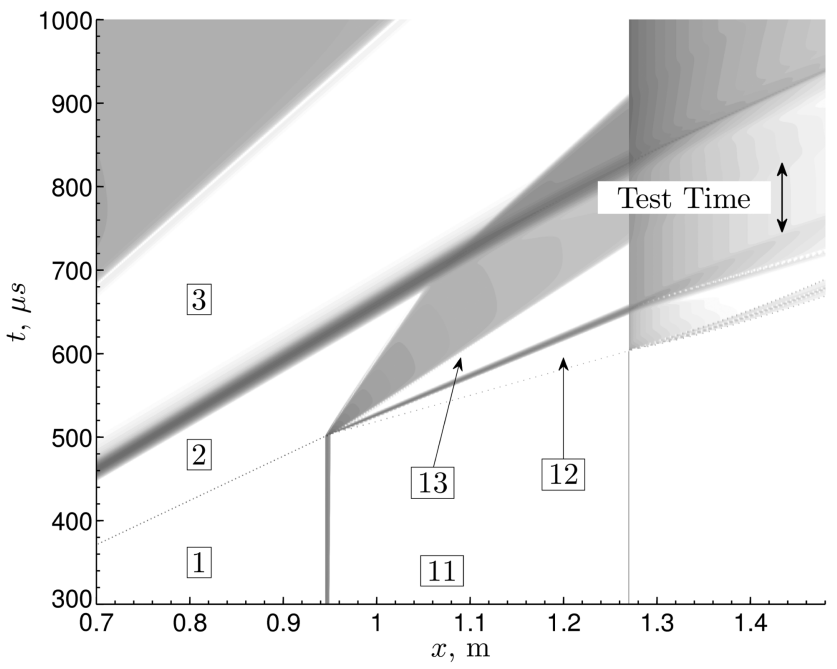

Fig. 8 Close-up of the accelerator section and nozzle from Fig. 7.

intermediate and accelerator sections. The sum of the intermediate and accelerator section lengths is still subject to the $L / d=50$ constraint. The same initial conditions as the proposed VET (Table 1) were used. A $10 \mathrm{deg}$ conical nozzle of length $L_{N}=0.16 \mathrm{~m}$ is used (area ratio of 10.7). Prior to the run, the nozzle is considered to be at a state similar to that of a dump tank, with air at low pressure ( $p_{N}=100 \mathrm{~Pa}, 750$ mTorr). A burst pressure of $30 \mathrm{kPa}$ is specified for the tertiary diaphragm. Until the diaphragm breaks, it acts as a perfectly reflecting, infinitely stiff wall. The primary diaphragm, the interface between the intermediate and accelerator sections, and the tertiary diaphragm are located at $0,0.94$, and $1.27 \mathrm{~m}$, respectively. The slightly larger length of the intermediate section when compared to a conventional ET $(0.94$ versus $0.86 \mathrm{~m})$ will be justified later.

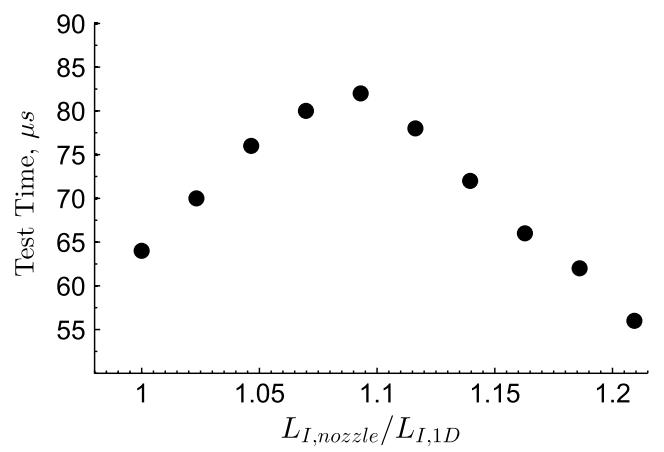

Fig. 9 Test time for varying lengths of the intermediate and accelerator sections when using a nozzle.

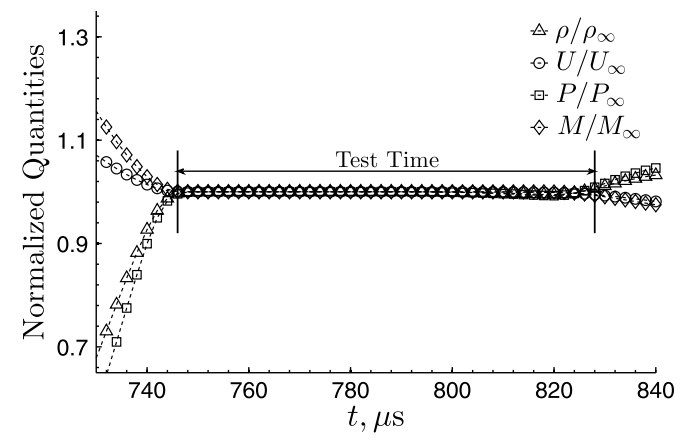

Fig. 10 Normalized flow conditions at a point $(x=1.43 \mathrm{~m})$ corresponding to an area ratio of 10.7 . 
Table 2 Comparison of run conditions available for a VET with varying fill pressures

\begin{tabular}{|c|c|c|c|c|c|c|c|c|c|c|c|c|}
\hline & $p_{4}(\mathrm{He}), \mathrm{MPa}$ & $p_{1}$ (air), $\mathrm{kPa}$ & $p_{11}(\mathrm{He}), \mathrm{kPa}$ & $h_{R}, \mathrm{MJ} / \mathrm{kg}$ & $p_{R}, \mathrm{MPa}$ & $T_{\text {Max }}, \mathrm{K}$ & $u_{\infty}, \mathrm{km} / \mathrm{s}$ & $p_{\infty}, \mathrm{kPa}$ & $\rho_{\infty}, \mathrm{kg} / \mathrm{m}^{3}$ & $T_{\infty}, \mathrm{K}$ & $M_{\infty}$ & $\tau, \mu \mathrm{s}$ \\
\hline VET (Table 1 ) & 8.16 & 7.75 & 7.75 & 3.8 & 2.4 & 2000 & 2.5 & 2.6 & 0.017 & 535 & 5.5 & 82 \\
\hline VET halfed & 8.16 & 3.88 & 3.88 & 4.5 & 1.5 & 2330 & 2.8 & 1.5 & 0.009 & 623 & 5.6 & 54 \\
\hline VET doubled & 8.16 & 15.5 & 15.5 & 3.1 & 3.6 & 1680 & 2.3 & 4.3 & 0.033 & 554 & 5.4 & 72 \\
\hline
\end{tabular}

Figures 7 and 8 show numerical $x$ - $t$ diagrams with the same fill pressures as the proposed VET (Table 1). An unsteady expansion is created when the secondary contact discontinuity enters the nozzle. The trailing characteristic from this unsteady expansion corresponds to the beginning of the test time. The test time is ended when either the tail or reflected head of secondary expansion wave reaches the test location. The qualitative behavior of the nozzle startup processes observed in Fig. $\underline{8}$ are consistent with the features seen in previous studies on nozzle startup phenomenon [36].

The predicted test time changes with the intermediate and accelerator lengths (Fig. 9). For all cases, the intermediate and accelerator section length sum is held constant at $1.27 \mathrm{~m} . L_{I, \text { nozzle }}$ refers to the length of the intermediate section when a nozzle is used, and this length is normalized by $L_{I, 1 D}$, the ideal intermediate section length when no nozzle is used $\left(L_{I, 1 D}=0.86 \mathrm{~m}\right)$. Increasing the intermediate section length (and reducing the accelerator section length), with respect to the 1-D case, increases the test time. After a certain threshold, the test time starts to decrease. All points to the left of the maximum test time (Fig. 9) are limited by the reflected secondary expansion wave head; all points to the right of the maximum test time are limited by the secondary expansion wave tail. The maximum point is where the tail and reflected head of the secondary expansion wave arrive at the test location inside the nozzle at the same time. A maximum test time of $80 \mu \mathrm{s}$ is found when $L_{I, \text { nozzle }}=0.94 \mathrm{~m}$ and $L_{A}=0.33 \mathrm{~m}$. Traces of $\gamma, \rho, p, u$, and $M$ are shown in Fig. 10 at the maximum test time. The average flow values, shown in Table 2 , are calculated during this test time at a downstream location of $x=\overline{1} .43 \mathrm{~m}$, which corresponds to an area ratio of 10.7 . In Table $2, p_{4}, p_{1}$ and $p_{11}$ are the initial pressures of the driver, intermediate, and accelerator sections, respectively; $h_{R}$ and $p_{R}$ are the effective reservoir mass specific enthalpy and pressure; $T_{\text {Max }}$ is the maximum temperature the test gas is raised to in the facility; $u_{\infty}, p_{\infty}$, $\rho_{\infty}, T_{\infty}$, and $M_{\infty}$ are the freestream velocity, pressure, density, temperature, and Mach number, respectively; and $\tau$ is the test time.

To show that it is possible to have more than one run condition for a given experimental setup, computations were performed with fill pressures in the intermediate and accelerator tubes doubled and halved from the originally proposed VET conditions (Table 2). The data are still sampled at a downstream location corresponding to an area ratio of 10.7 (fixed nozzle length). It is important to note that the maximum static temperature reached in the half-pressure trial is approximately $2350 \mathrm{~K}$, which means that the perfect-gas assumption will begin to break down, and so the calculated properties are presented as an estimate. Nonetheless, useful test times and test conditions are still generated when the fill conditions of the VET are changed.

\section{Initial Test Gas Accelerator Gas Separation}

We propose that the intermediate and accelerator chambers are to be initially separated by a fast-acting sliding valve. This sliding valve will separate the two tubes during filling and then be pulled away before the primary diaphragm is burst. The design of such a sliding valve would be similar to one that has been shown to work for detonation-gas interface studies in GALCIT's Detonation Tube $[48,49]$, or a similar separation strategy used in vertical shock tube studies $[50,51]$. The helium in the accelerator tube will diffuse into the intermediate tube after the sliding valve is pulled away. A characteristic diffusion length scale $L_{\text {diff }}$ associated with helium and air can be approximated as $L_{\text {diff }} \approx \sqrt{D t_{\text {diff }}}$, where $D$ is the binary diffusion coefficient for air and helium, and $t_{\text {diff }}$ is a characteristic time. The characteristic diffusion time is chosen to be $50 \mathrm{~ms}$, which is an estimate of the sliding valve opening time. For practical tube fill pressures $\left(p_{1}, p_{11} \approx 10 \mathrm{kPa}\right), D \approx 0.001 \mathrm{~m}^{2} / \mathrm{s}[29,52]$, which gives a diffusion distance of about $L_{\text {diff }}=7 \mathrm{~mm}$ in $t_{\text {diff }}=50 \mathrm{~ms}$. This is an acceptable diffusion length and opening time.

\section{Conclusions}

In this work, we propose a test facility configuration termed the vertical expansion tunnel (VET). This facility avoids the detrimental effects of secondary diaphragm rupture in a conventional ET by eliminating its necessity. The secondary diaphragm is no longer required if the facility is oriented vertically and the intermediate and accelerator tubes are filled to the same initial pressure with gases of different density, with light gases located over heavy gases (e.g., helium over air) so that the interface is hydrodynamically stable. The intermediate/accelerator interface would be created just before the run by a fast-acting sliding valve that will be removed before the bursting of the primary diaphragm. The sliding valve opening time and characteristic diffusion length of the helium into the test gas are shown to be acceptable. The maximum reservoir mass specific enthalpy and pressure of the VET are higher than for an RST but below the ET values. Perfect-gas quasi-one-dimensional Euler computations and performance estimates indicate that the test time in the VET is slightly longer than in the ET but shorter than in the RST. Increasing the test time of the facility would require an increase in length, noting that the test time scales approximately linearly with overall length. In doing so, the machine would not fit into a singlestory laboratory, and a multi-story design, like that successfully used in vertical shock tubes $[\underline{50,51]}$, would be required. If some sacrifice of the effective reservoir conditions can be made, the VET could be used in hypervelocity ground testing to eliminate problems associated with secondary diaphragm rupture while maintaining useful performance.

\section{Acknowledgments}

The authors would also like to thank Siddhartha Verma for his contribution to the work. This work was an activity that was part of National Center for Hypersonic Laminar-Turbulent Research, sponsored by the "Integrated Theoretical, Computational, and Experimental Studies for Transition Estimation and Control" project supported by the U.S. Air Force Office of Scientific Research and the National Aeronautics and Space Administration (FA9552-09-10341). The views and conclusions contained herein are those of the authors and should not be interpreted as necessarily representing the official policies or endorsements, either expressed or implied, of the U.S. Air Force Office of Scientific Research or the U.S. Government.

\section{References}

[1] Trimpi, R. L., "A Preliminary Theoretical Study of The Expansion Tube, a New Device for Producing High-Enthalpy Short-Duration Hypersonic Gas Flows," NASA TR-R-133, 1962.

[2] Trimpi, R. L., and Callis, L. B., "A Perfect-Gas Analysis of the Expansion Tunnel, a Modification to the Expansion Tube," NASA TRR-223, 1965.

[3] Lukasiewicz, J., Experimental Methods of Hypersonics, 1st ed., Marcel Decker, New York, 1973, pp. 169-206.

[4] Ben-Yakar, A., and Hanson, R. K., "Characterization of Expansion Tube Flows for Hypervelocity Combustion Studies," Journal of Propulsion and Power, Vol. 18, No. 4, 2002, pp. 943-952. doi: $10.2514 / 2.6021$

[5] Norfleet, G. D., Lacey, J. J., and Whitfield, J. D., "Results of an Experimental Investigation of the Performance of an Expansion Tube," 
Proceedings of the 4th Hypervelocity Techniques Symposium, Ballistic Research Labs., Aberdeen Proving Ground, MD, 1965, pp. 49-110.

[6] Spurk, J. H., "Design, Operation and Preliminary Results of the BRL Expansion Tube," Proceedings of the 4th Hypervelocity Techniques Symposium, Ballistic Research Labs., Aberdeen Proving Ground, MD, 1965, pp. 114-144.

[7] Miller, C. G., "Operational Experience in the Langley Expansion Tube with Various Gases," NASA TM-78637, 1977.

[8] Shinn, J. L., and Miller, C. G., "Experimental Perfect-Gas Study of Expansion-Tube Flow Characteristics," NASA TP-1317, 1978.

[9] Paull, A., and Stalker, R. J., "The Effect on an Acoustic Wave as It Traverses an Unsteady Expansion," Physics of Fluids, Vol. 3, No. 4, 1991, pp. 717-719. doi:10.1063/1.858079

[10] Paull, A., and Stalker, R. J., "Test Flow Disturbances in an Expansion Tube," Journal of Fluid Mechanics, Vol. 245, 1992, pp. 493-521. doi:10.1017/S0022112092000569

[11] Jacobs, P., "Numerical Simulation of Transient Hypervelocity Flow in an Expansion Tube," Computers and Fluids, Vol. 23, No. 1, 1994, pp. $77-101$. doi:10.1016/0045-7930(94)90028-0

[12] Dufrene, A., Sharma, M., and Austin, J. M., "Design and Characterization of a Hypervelocity Expansion Tube Facility," Journal of Spacecraft and Rockets, Vol. 23, No. 6, 2007, pp. 1185-1193. doi: $10.2514 / 1.30349$

[13] Hornung, H. G., "Experimental Hypervelocity Flow Simulation, Needs, Achievements and Limitations," Proceedings of the 1st Pacific International Conference on Aerospace Science and Technology, National Cheng Kung University, Tainan, Taiwan, 1993.

[14] Bakos, R. J., and Morgan, R. G., "Chemical Recombination in an Expansion Tube," AIAA Journal, Vol. 32, No. 6, 1994, pp. 1316-1319. doi: $10.2514 / 3.12135$

[15] Payman, W., and Shepherd, W. C. F., "Explosion Waves and Shock Waves VI. The Disturbance Produced By Bursting Diaphragms with Compressed Air," Proceedings of the Royal Society of London Series A: Mathematical and Physical Sciences, Vol. 186, No. 1006, 1946, pp. 293-321.

[16] White, D. R., "Influence of Diaphragm Opening Time on ShockTube Flows," Journal of Fluid Mechanics, Vol. 4, No. 6, 1958, pp. 585599. doi: $10.1017 / \mathrm{S} 0022112058000677$

[17] Gaydon, A. G., and Hurle, I. R., The Shock Tube in High-Temperature Chemical Physics, 1st ed., Chapman \& Hall, London, 1963, pp. 83-107.

[18] Simpson, C. J., Chandler, T. R., and Bridgman, K. B., "Effect on Shock Trajectory of the Opening Time of Diaphragms in a Shock Tube," Physics of Fluids, Vol. 10, No. 9, 1967, pp. 1894-1897. doi:10.1063/1.1762384

[19] Rothkopf, E. M., and Low, W., "Diaphragm Opening Process in Shock Tubes," Physics of Fluids, Vol. 17, No. 6, 1974, pp. 1169-1173. doi:10.1063/1.1694860

[20] Roberts, G. T., Morgan, R. G., and Stalker, R. J., "Influence of Secondary Diaphragm on Flow Quality in Expansion Tubes," Proceedings of the 19th International Symposium on Shock Waves, Springer-Verlag, Pasadena, CA, 1995, pp. 203-208.

[21] Wegener, M., Sutcliffe, M., and Morgan, R., "Optical Study of a Light Diaphragm Rupture Process in an Expansion Tube," Shock Waves, Vol. 10, No. 3, 2000, pp. 167-178. doi: $10.1007 / \mathrm{s} 001930050003$

[22] Furukawa, T., Aochi, T., and Sasoh, A., "Expansion Tube Operation with Thin Secondary Diaphragm," AIAA Journal, Vol. 45, No. 1, 2007, pp. 214-217. doi: $10.2514 / 1.23846$

[23] Neely, A. J., and Morgan, R. G., "The Superorbital Expansion Tube Concept, Experiment and Analysis," Aeronautical Journal, Vol. 98, No. 973,1994 , pp. 97-105.

[24] Erdos, J. I., Bakos, R. J., Castrogiovanni, A., and Rodgers, R. C., "Dual Mode Shock-Expansion/Reflected-Shock Tunnel," AIAA Paper $1997-$ 0560, Jan. 1997.

[25] Sasoh, A., Ohnishi, Y., Ramjaun, D., Takayama, K., Otsu, H., and Abe, T., "Effective Test Time Evaluation in High-Enthalpy Expansion Tube," AIAA Journal, Vol. 39, No. 11, 2001, pp. 2141-2147. doi: $10.2514 / 2.1210$

[26] Heltsley, W. N., Snyder, J. A., Houle, A. J., Davidson, D. F., Mungal, M. G., and Hanson, R. K., "Design and Characterization of the Stanford 6 Inch Expansion Tube," AIAA Paper 2006-4443, July 2006.

[27] Holden, M. S., Wadhams, T. P., and Candler, G. V., "Experimental Studies in the LENS Shock Tunnel and Expansion Tunnel to Examine Real-Gas Effects in Hypervelocity Flows," AIAA Paper 2004-0916, Jan. 2004.
[28] Liepmann, H. W., and Roshko, A., Elements of Gasdynamics, Dover, Mineloa, New York, 1993, pp. 74-83.

[29] Goodwin, D. G., "An Open-Source, Extensible Software Suite for CVD Process Simulation," Electrochemical Society, Vol. 14, Proceedings of Electrochemical Society (ECS), Pennington, New Jersey, 2003, pp. 2003-2008.

[30] Browne, S., Ziegler, J., and Shepherd, J. E., "Numerical Solution Methods for Shock and Detonation Jump Conditions," Graduate Aerospace Lab. of the California Inst. of Technology, Rept. FM2006006, Pasadena, CA, 2006

[31] Gordon, S., and McBride, B., "Thermodynamic Data to $20000 \mathrm{~K}$ for Monatomic Gases," NASA TP-1999-208523, 1999.

[32] McBride, B., Zehe, M. J., and Gordon, S., "NASA Glenn Coefficients for Calculating Thermodynamic Properties of Individual Species," NASA TP-2002-211556, 2002.

[33] Gupta, R. N., Yos, J. M., Thompson, R. A., and Lee, K. P., “A Review of Reaction Rates and Thermodynamic and Transport Properties for an 11Species Air Model for Chemical and Thermal Nonequilibrium Calculations to $30000 \mathrm{~K}$," NASA RP-1232, 1990.

[34] Kao, S., and Shepherd, J. E., "Numerical Solution Methods for Control Volume Explosions and ZND Detonation Structure," Graduate Aerospace Lab. of the California Inst. of Technology, Rept. FM2006007, Pasadena, CA, 2006.

[35] Mirels, H., "Shock Tube Test Time Limitation Due to Turbulent-Wall Boundary Layer," AIAA Journal, Vol. 2, No. 1, 1964, pp. 84-93.

[36] Smith, C. E., "The Starting Process in a Hypersonic Nozzle," Journal of Fluid Mechanics, Vol. 24, 1966, pp. 625-640. doi: $10.1017 / \mathrm{S} 0022112066000880$

[37] Davies, L., and Wilson, J. L., "Influence of Reflected Shock and Boundary-Layer Interaction on Shock-Tube Flows," Physics of Fluids, Vol. 12, No. 5, 1969, pp. 37-43. doi:10.1063/1.1692625

[38] Sudani, N., and Hornung, H. G., "Gasdynamical Detectors of Driver Gas Contamination in a High-Enthalpy Shock Tunnel," AIAA Journal, Vol. 36, No. 3, 1998, pp. 313-319. doi: $10.2514 / 2.383$

[39] Wintenberger, E., Austin, J. M., Cooper, M., Jackson, S., and Shepherd, J. E., "Analytical Model for the Impulse of Single-Cycle Pulse Detonation Tube," Journal of Propulsion and Power, Vol. 19, No. 1, 2003, pp. 22-38. doi: $10.2514 / 2.6099$

[40] Glaister, P., "Flux Difference Splitting for the Euler Equations in One Spatial Coordinate with Area Variation," International Journal for Numerical Methods in Fluids, Vol. 8, No. 1, 1988, pp. 97-119.

[41] Roe, P. L., "Approximate Riemann Solvers, Parameter Vectors, and Difference Schemes," Journal of Computational Physics, Vol. 43, No. 2, 1981, pp. 357-372. doi:10.1016/0021-9991(81)90128-5

[42] Leveque, R. J., Finite Volume Methods for Hyperbolic Problems, Cambridge Texts in Applied Mathematics, Cambridge Univ. Press, Cambridge, England, U.K., 2007, pp. 181-183.

[43] Van Leer, B., "Towards the Ultimate Conservative Difference Scheme 2. Monotonicity and Conservation Combined in a Second Order Scheme," Journal of Computational Physics, Vol. 14, No. 4, 1974, pp. 361-370. doi:10.1016/0021-9991(74)90019-9

[44] Lax, P. D., and Liu, X.-D., "Solution of Two-Dimensional Riemann Problems of Gas Dynamics by Positive Schemes," SIAM Journal on Scientific Computings, Vol. 2, No. 2, 1998, pp. 319-340. doi:10.1137/S1064827595291819

[45] Sanders, R., Morano, E., and Druget, M.-C., "Multidimensional Dissipation for Upwind Schemes: Stability and Applications to Gas Dynamics," Journal of Computational Physics, Vol. 145, No. 2, 1998, pp. 511-537. doi:10.1006/jcph.1998.6047

[46] Abgrall, R., and Karni, S., "Computations of Compressible Multifluids," Journal of Computational Physics, Vol. 169, No. 2, 2001, pp. 594-623. doi: $10.1006 / \mathrm{jcph} .2000 .6685$

[47] Purser, R. J., and Leslie, L. M., "An Efficient Interpolation Procedure for High-Order Three-Dimensional Semi-Lagrangian Models," Monthly Weather Review, Vol. 119, No. 10, 1991, pp. 2492-2498. doi:10.1175/1520-0493(1991)119<2492:AEIPFH>2.0.CO;2

[48] Lieberman, D. H., Detonation Interaction with Sharp and Diffuse Interfaces, Ph.D. Thesis, California Inst. of Technology, Pasadena, CA, 2006.

[49] Lieberman, D. H., and Shepherd, J. E., "Detonation Interaction with a Diffuse Interface and Subsequent Chemical Reaction," Shock Waves, Vol. 16, No. 6, 2007, pp. 421-429. doi:10.1007/s00193-007-0080-3 
[50] Brouillette, M., and Sturtevant, B., "Growth Induced by Multiple Shock Waves Normally Incident on Plane Gaseous Interfaces," Physica D: Nonlinear Phenomena, Vol. 37, Nos. 1-3, 1989, pp. 248-263. doi:10.1016/0167-2789(89)90133-4

[51] Anderson, M. H., Puranik, B. P., Oakley, J. G., Brooks, P. W., and Bonazza, R., "Shock Tube Investigation of Hydrodynamic Issues Related to Inertial Confinement Fusion," Shock Waves, Vol. 10, No. 5,
2000, pp. 377-387.

doi: $10.1007 / \mathrm{s} 001930000067$

[52] Hirschfelder, J. O., Curtiss, C. F., and Bird, R. B., Molecular Theory of Gases and Liquids, Wiley, New York, 1954, pp. 478-480.

F. Alvi Associate Editor 